International Journal of Engineering \& Technology, $7(2.24)(2018) 159-165$
International Journal of Engineering \& Technology
SPC
Website: www.sciencepubco.com/index.php/IJET
Research paper

\title{
Orientation Feature Transform Model for Image Retrieval in Sketch Based Image Retrieval System
}

\author{
Durga Prasad K ${ }^{1 *}$, Manjunathachari K², Giri Prasad M.N ${ }^{3}$ \\ ${ }^{1}$ Research scholar, Department of ECE, JNTUA, Ananthapuramu, A.P, India \\ ${ }^{2}$ Professor and HOD, Department of ECE, GITAM University Hyderabad, Telangana, India \\ ${ }^{3}$ Professor, Department of ECE, JNTUA college of Engineering, Ananthapuramu, A.P, India \\ *Corresponding Author E-mail: durga3139@gmail.com
}

\begin{abstract}
This paper focus on Image retrieval using Sketch based image retrieval system. The low complexity model for image representation has given the sketch based image retrieval (SBIR) a optimal selection for next generation application in low resource environment. The SBIR approach uses the geometrical region representation to describe the feature and utilize for recognition. In the SBIR model, the features represented define the image. Towards the improvement of SBIR recognition performance, in this paper a new invariant modeling using "orientation feature transformed modeling" is proposed. The approach gives the enhancement of invariant property and retrieval performance improvement in transformed domain. The experimental results illustrate the significance of invariant orientation feature representation in SBIR over the conventional models.
\end{abstract}

Keywords: Sketch Based Image Retrieval, Orientation Feature Transformed Modeling.

\section{Introduction}

Images are used as a source of information representation in current technology. With evolution of new technologies, images has become a source of data exchange where, the image data are used as a authentication means, critical data exchange, information exchange, photography etc. The new technology has evaluated into advanced mean of data storage and retrieval, such as the cloud network and distrusted data computing. With the increase in data storage and integration of distributed resources, the information retrieval has gained a large knowledge of details, which are constraint at the primitive level. However, with the increase in image information, the processing overhead has proportionally increased, leading to system overhead and slow retrieval performance. It is hence needed to develop new approach in image retrieval for faster and accurate retrieval under resource constraint environments. In the effort for faster image retrieval sketch based image retrieval has come out as one of the prominent solution. Shape based image retrieval has gained a lot interest in recent past. The feature extraction were developed based on the global feature extraction, where global feature such as the invariant moments [1], shape feature[2], and combined feature of shape and invariant moments using histogram descriptor and angular radial transform (ART) [3] were developed. The features are predominantly been used in image and video coding for information retrieval. In the development of sketch based information retrieval (SBIR), different approaches were proposed in past. In [4] a distance transform approach for image retrieval based on the distance vector for a query sample and the natural image is developed. The features are transformed to a high dimensional binary hash pattern to represent the shape feature.
In [5] the approach of SBIR for information exchange for communication is presented. New sketch patterns were exchanged in the information exchange in common communication. The approach of SBIR using a shape and texture feature for a given query in developed in [6]. The approach has defined the utilization of texture shape, color for the SBIR operation. In the utilization of SBIR based application for real time application, in [7] a songket motive technique for repository system is defined. The proposed approach defined an approach for sketch based information retrieval in repository information retrieval. In [8] a fine grain sketch based information retrieval is defined. The expressiveness in representing the sketch is focused. For a large scale approach in $[9,10]$ a simple and efficient SBIR system is presented. The challenge of localization of image region in such application is defined. The approach defined a contour method for the retrieval process. In [11] a hand drawn sketch method for SBIR system is proposed. The shape retrieval features are defined based on the edge parameter, where a hand drawn sketch is passed to an edge descriptor and the edge regions are used as a feature descriptor in information retrieval. The web based approach for SBIR is outlined in [12]. The approach defines a web browser based retrieval approach where the image is passed as an interface of hand drawn sketch for manga image titles. In [13] fine grain retrieval for SBIR application (FG-SBIR) is presented. the FGSBIR is a new approach for SBIR application, where the sketch image are processed in finer scale level to extract finer details of the curvature contours presented in the sketch regions. The past development of SBIR application is summarized in [14]. The limitations of SBIR in information retrieval is well outlined in [15, 16], where the operational comparison of image retrieval based on the sketch and image retrieval were compared. 
Wherein approaches were developed to derive feature vector for image retrieval, the issue of invariance exist which degrades the retrieval performance. The description feature in the transformed domain would lead to higher spectral details leading to accurate feature representation for image retrieval. In this paper to obtain a invariance property in SBIR and to achieve a higher degree of feature representation, a new SBIR model using orientation feature transformed (OFT) is proposed. To outline the suggested approach, this paper is outlined in 6 sections, wherein section 2 defines the approach of a SBIR model; section 3 outlines the proposed approach of orientation feature transformed modeling. Section 4 illustrates the obtained observation for the developed systems with the conclusion derived in section 5 .

\section{Sketch Based Image Retrieval}

Retrieving the relevant information from keywords is very major task and this technique has been focused by researchers in many ways. Sketch is one format of language exchange used in various applications, and one of the strong media for communication in old era as well as in current scenarios. Hence currently sketch based image retrieval (SBIR) is also in focus for research purpose. Existing system descriptor SBIR is derived from two classic descriptor namely, SHoG and GF-HOG. The shape descriptors used in the feature extraction are based on image patches or line descriptors. In the image patch, based approach the isolation of an edge region is based in the supportive neighbor pixels and the extractions are difficult under different semantic regions. Lime based descriptors are much simpler, but the approach is limited to noise effect and result in misclassified feature details. A basic SBIR model for information retrieval is illustrated in Fig. 1.

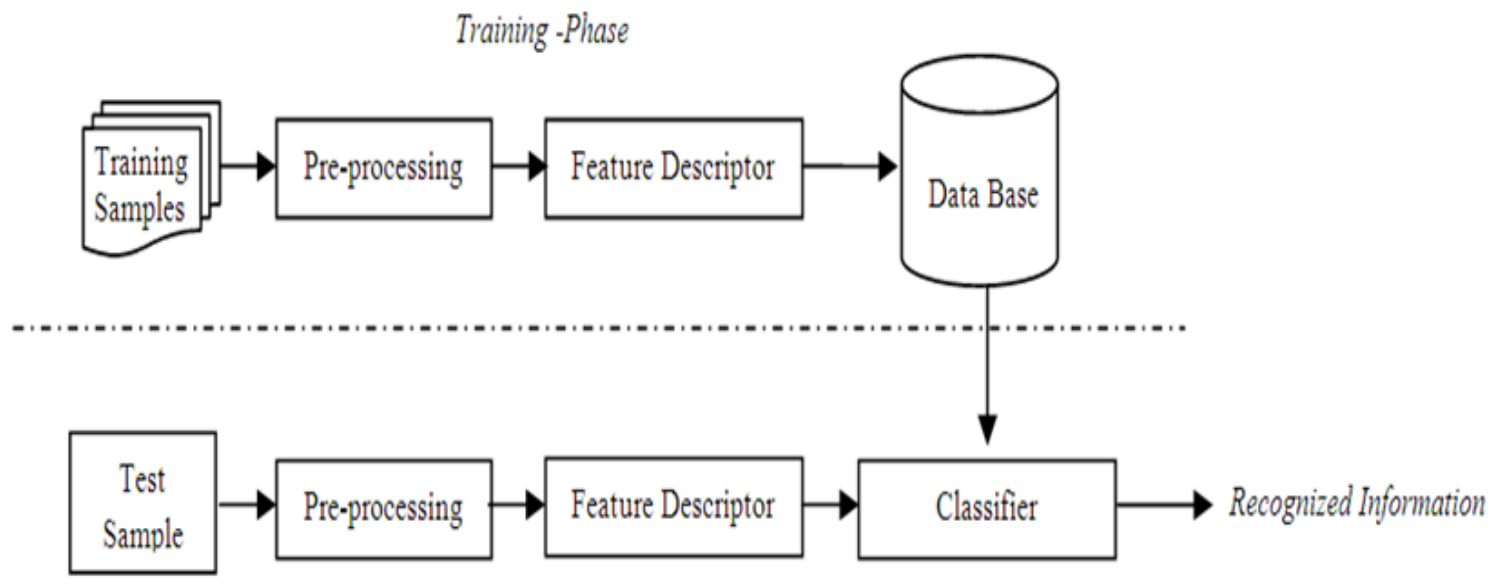

Testing -Phase

Fig. 1: A SBIR Frame work for information retrieval

The propose system has two operational stages of training and testing. Wherein training phase is used to train the learning system, testing phase is used to test a given query sketch for information retrieval. The training process is performed to train the learning system with different shape features of different objects which is considerable in retrieval stage. The testing process is processed with a classifier unit, which performs a nearest matching for the retrieval of information from the trained database. The classifier is designed with a Euclidian distance based approach to obtain the best set of matches from the knowledge dataset. The decision ' $\mathrm{D}$ ' for the retrieval is derived as the minimum value of the Euclidian distance defined as,

$D=\min \left(E d_{i}\right)$

Where,

Euclidian Distance, $E d_{i}=\sqrt{\sum_{i=1}^{M} F_{q}-F d b_{i}}$ (2)

Where, $F_{q}$ is the feature describing for query and,

$F d b_{i}$ is the features trained during the learning process of training system.

The SBIR system defines the image feature based on the geometrical feature representation of contour and lines feature to extract image details. In the sketch based information retrieval, invariant features and gradient model were used in past. The SBIR feature extraction uses the basic local appearance and extracts the edge region based on the intensity distribution in an image. In the process of edge descriptor, for a large image region, images are blocked into small regions of uniform dimensions and each of the regions is processed for intensity mapping for edge region prediction based on energy distribution at the bounding regions. The approach result in defining the bounding edge regions, however, the suggested approach has a limitation of redundant feature vector in different orientations, as the scale invariant feature are measured in different orientation, the redundant features exist which are processed multiple time giving processing overhead. it is hence required to minimize the processing recursion. An orientation feature transform modeling suggested in following section outline the suggested approach.

\section{Orientation Feature Transformed Modeling (OFT)}

In the feature representation, the given image is transformed into frequency domain to get a frequency resolution representation. In this paper, the image is transformed using Gabor transformation, which gives the advantage of scaling, orientation invariance property. Gabor transform were proposed Gabor transformation is used as a mean of extraction of directional and linear components for a image feature description. The transformation defines the Gabor kernel to extract the feature of each of the decomposed direction and extract the feature based on the orientation, and scaling for invariance in feature extraction. The feature extraction in each of this sub decomposed image region is developed using orientations feature extraction and scaling parameter.

In the transformation process of Gabor operation, the kernel is defined as sinusoid of the Gaussian envelop with the standard deviation of each of the image region. The decomposition of the image for feature extraction is defined using a threshold limit. The kernel of Gabor function is defined in equation (3).

$\psi(i, j)=\frac{1}{2 \pi \sigma_{i} \sigma_{j}} \exp \left[-\frac{1}{2}\left(\frac{i^{2}}{\sigma_{i}^{2}}+\frac{j^{2}}{\sigma_{j}^{2}}\right)+2 \pi j W i\right]$ 
The Gabor function is normalized to a DC level to give a low redundancy in the feature representation for different dilation and rotation effect of $\psi(i, j)$ defined by (3). The transformed frequency domain of the Gabor kernel is given by,

$\Psi(k, l)=\frac{1}{2 \pi \sigma_{k} \sigma_{l}} \exp \left\{-\frac{1}{2}\left[\frac{(k-W)^{2}}{\sigma_{k}^{2}}+\frac{l^{2}}{\sigma_{l}^{2}}\right]\right\}$

In the proposed approach, the transformation is used to define a minimal of half peak region to define the intensity variation of image bounding region at the edge varying regions in the image The process is to minimize the distortional effects observed at the edge regions and the error observed due to free hand sketch drawing. The features are derived to minimize the redundancy pattern elimination in training and minimize the testing overhead by selecting distinct feature vectors. The obtained output for the Gabor transform at different orientation reveals the orientation in one particular direction, an average Gabor output is derived given by,

$G_{\text {avg }}=\left|\sum I(i, j)\right|$

Where $I(i, j)$ is the gabor output obtained at each orientation.

To extract the line regions, a logical ANDing operation over obtained eight orientations were carried out, where each orientation is transformed to a bi-level logic using global thresholding as illustrated. In the threshold process, the image is sub divided into two regions of required and non-required regions. In the isolation of the regions, the image is threshold based on the image pixel values, defining the intensity of the image and resolution component. In the process of threshold evaluation, the image is processed with its neighbor pixel content to define a targeted threshold value. The threshold is treated as a line margin for the image in reference to extract the region of interest based on the pixel intensity. The threshold processes removes the non-linear segmented regions and extract the non-linear component of the image region of processing. In the extraction of the threshold value, a $3 \times 3$ neighbor search method is used, w here each of the pixel is processed taking as a reference, and a $3 \times 3$ neighbor search is made to derive the threshold value. the mask operator used in the process is a $3 \times 3$ ones matrix with all the element sets to value of ' 1 ' when to be traced in that direction. For a given image region ' $r$ ' the threshold is derived by,
$T h=\frac{\sum\left(r_{m}(x, y) X m(x, y)\right)}{\sum m(x, y)}$

Where,

$m(i, j)=\max \left(\left|K_{1} * * r_{m}(i, j)\right|,\left|K_{2} * * r_{m}(i, j)\right|\right)$

and,

$K_{1}=\left[\begin{array}{lll}-1 & 0 & 1\end{array}\right], K_{2}=\left[\begin{array}{lll}-1 & 0 & 1\end{array}\right]^{t}$

The threshold regions are divided into a high and a low intensity image region, where a high values used for region of interest and a low value is assigned for a non-interested region. The regions of interest in this case are the segmenting region of sketch input. To extract the hand drawn sketch, during the process edge information elimination is observed. To recover the edge back, morphological operator is used to define the edge region. A logical AND operation is used with morphological operation to perform the region extraction and mapping operation.

A co-occurrence pattern is extracted to derive the region of interest, where the image is processed to derive the affine feature extraction in the image. The redundant pattern of the edge region in this case is developed in a pixel pair-wise manner. The patterns were observed to be varying with finer to coarse feature in this matrix. To derive the distance between the pixels, and degree of pixel variation co-occurrence feature mapping is used. the computation process of the measurement is depicted in Fig. 2.

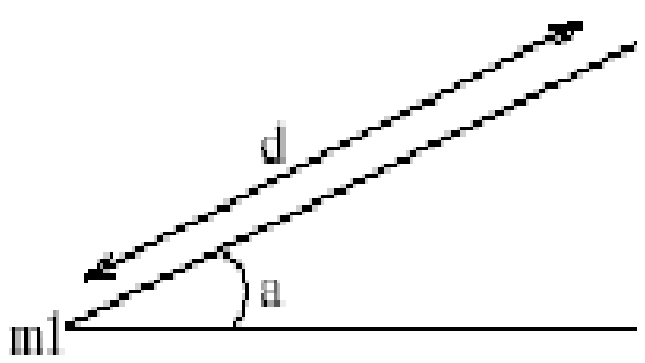

m2

Fig. 2: Distance and degree of variation in the image pixels

The algorithm to construct a co-occurrence matrix is depicted below.

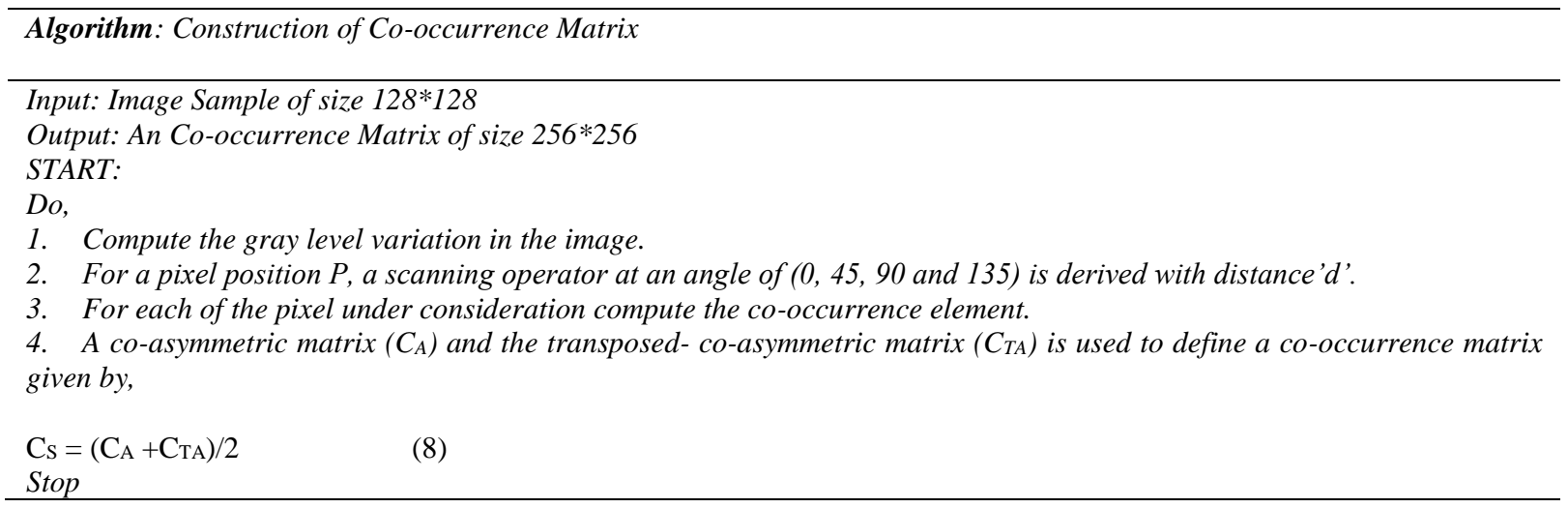

\section{Experimental Result}

To validate the proposed approach, a simulation model on Matlab tool using image processing toolbox is developed. The results obtained, are as outlined below. The proposed approach is evaluated over coil-20 database. Few samples of this data set is as shown in Fig. 3. 


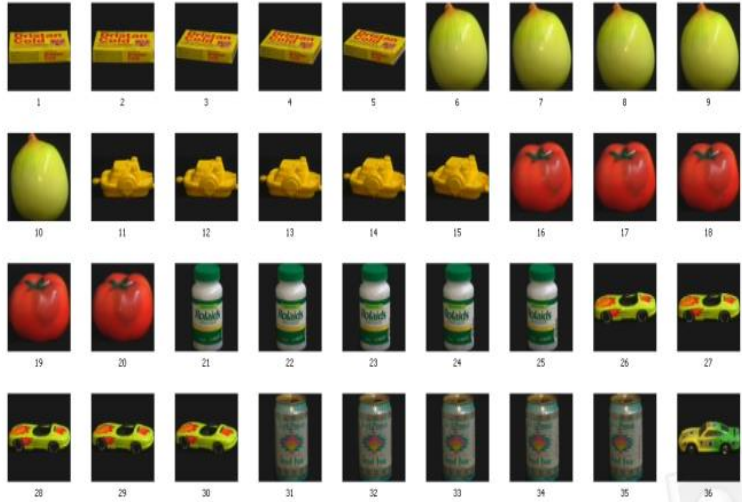

Fig. 3: Database samples from coil-100 Dataset.

To evaluate the processing process of natural features the test sample is passed with a different advanced system direction. Notes from a sophisticated system below,

Sample I: Sample with $0^{0}$ orientation

\section{Query image}

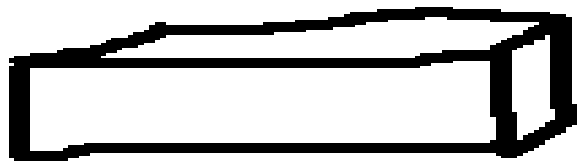

Fig. 4: Original Query sample at $0^{0}$ orientation

A selected test sample for the recognition process is shown in Fig. 4.
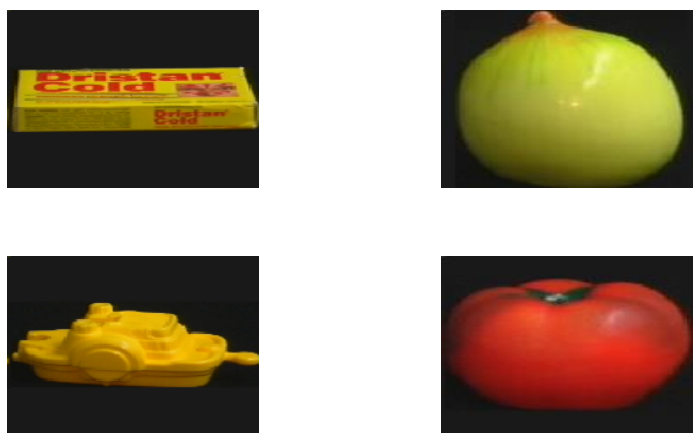

Fig. 5: Top 4 classified sample using OFT
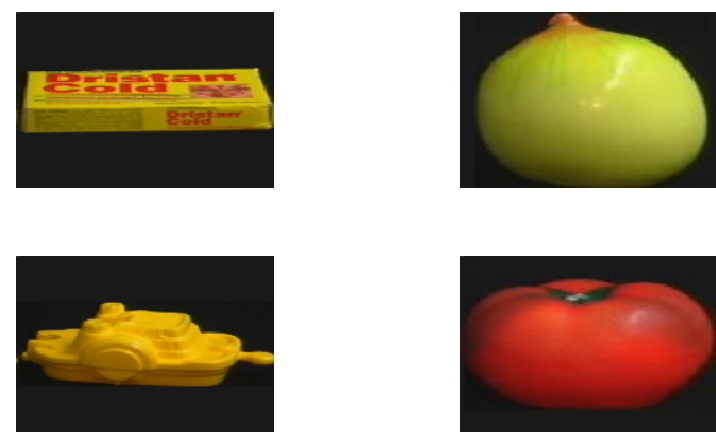

Fig. 6: Top 4 classified sample using SIFT-HOG

The top 4 classified sample from the trained data base for the proposed OFT and SIFT-HOG feature descriptor is shown in Fig. 5 and 6 respectively.

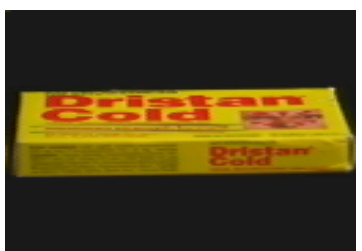

(a)

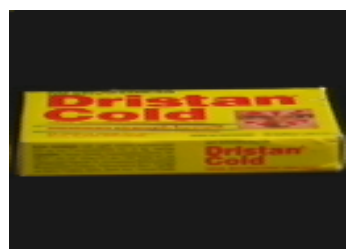

(b)
Fig. 7: Final retrieved sample (a) Using OFT (b) SIFT-HOG

The top retrieved sample from the data base after classification for the two methods is shown in Fig. 7 (a) and (b) respectively. This test sample is then evaluated over different orientation and the obtained results are as illustrated below;

Sample II: Sample with $15^{0}$ orientation

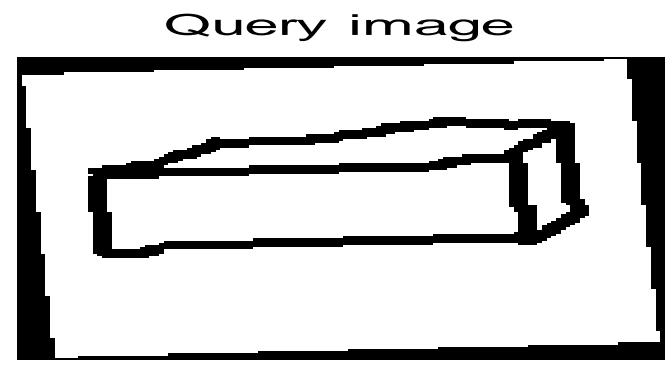

Fig. 8: Query sample at orientation of $15^{\circ}$

The test sample is oriented by $15^{\circ}$ orientation, and passed as a query sample to the developed system. The features are extracted using conventional SIFT-HOG features and OFT features. Using theses feature coefficients; recognition process is carried out using K-NN classifier. The obtained results of the classified observations are illustrated in Fig. 9.
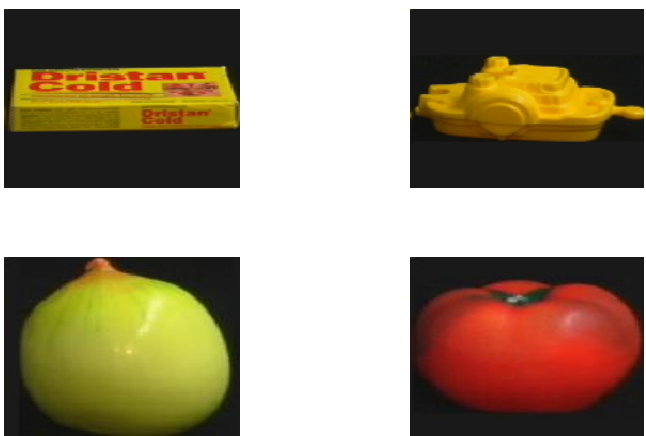

Fig. 9: Top 4 classified sample using OFT

When applied with orientation it is observed that the features are extracted from the OFT coefficients hence the feature variation due to spectral orientation is not effective, as these coefficients are scaled to a uniform scale. However this variation is retained in SIFT-HOG based feature descriptor hence the classification process is affected. 

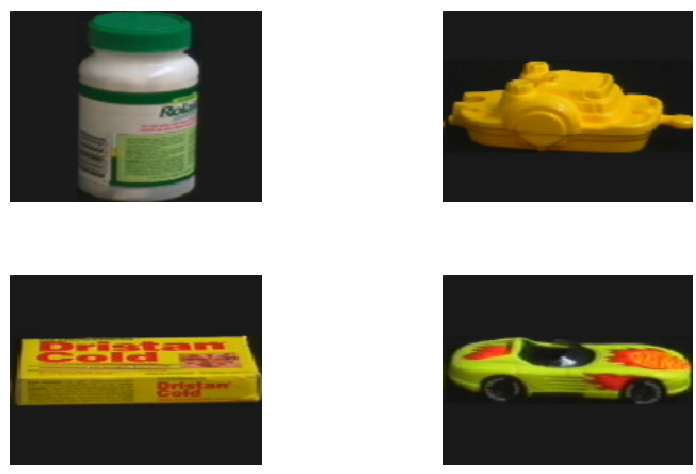

Fig. 10: Top 4 classified sample using SIFT-HOG

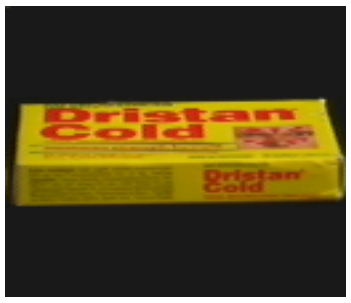

(a)

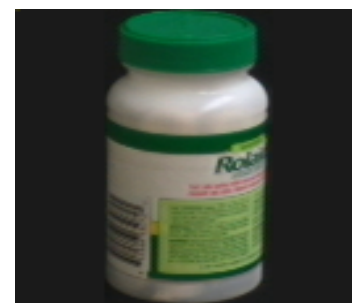

(b)
Fig. 11: Final retrieved sample (a) Using OFT (b) SIFT-HOG Sample III: Sample with $45^{\circ}$ orientations

\section{Query image}

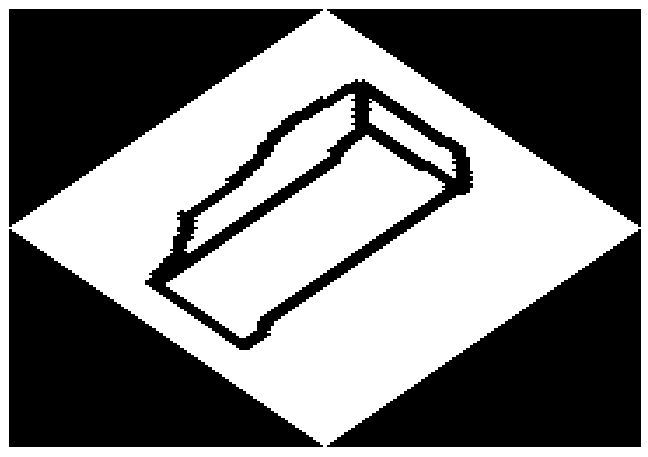

Fig. 12: Query sample at $45^{\circ}$ orientation
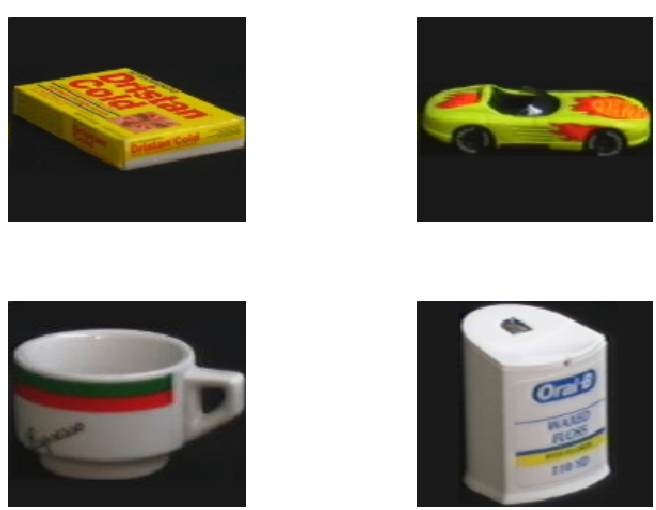

Fig. 13: Top 4 classified sample using OFT
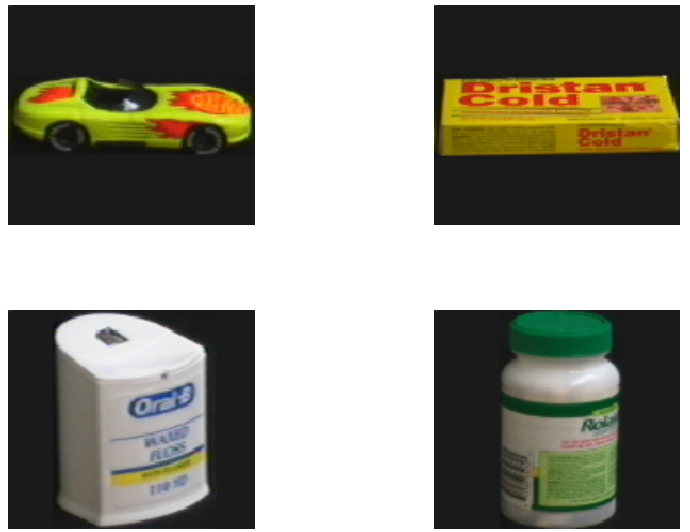

Fig. 14: Top 4 classified sample using SIFT-HOG

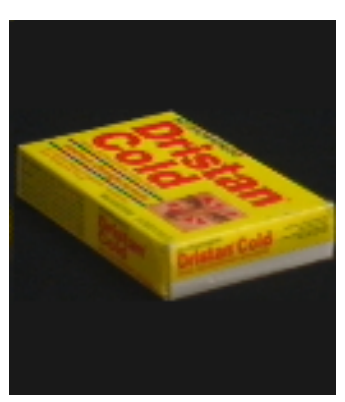

(a)

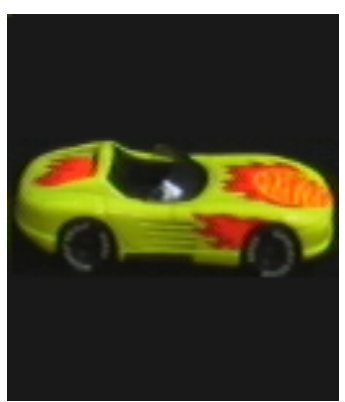

(b)
Fig. 15: Final retrieved sample (a) Using OFT (b) SIFT-HOG

Analysis of different test samples is carried out in different directions. The test results of these samples are described in Fig. 15 .

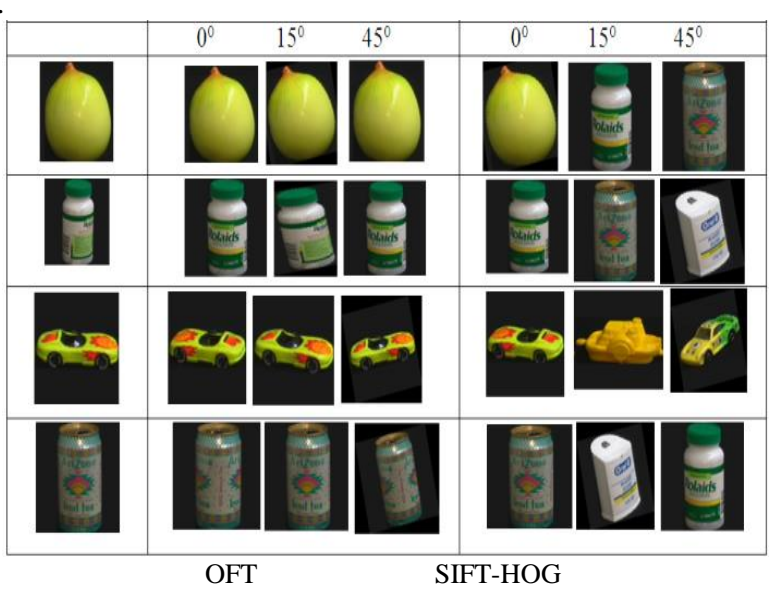

Fig. 16: observations obtained for different test samples at 0,15 , $45^{\circ}$ orientations

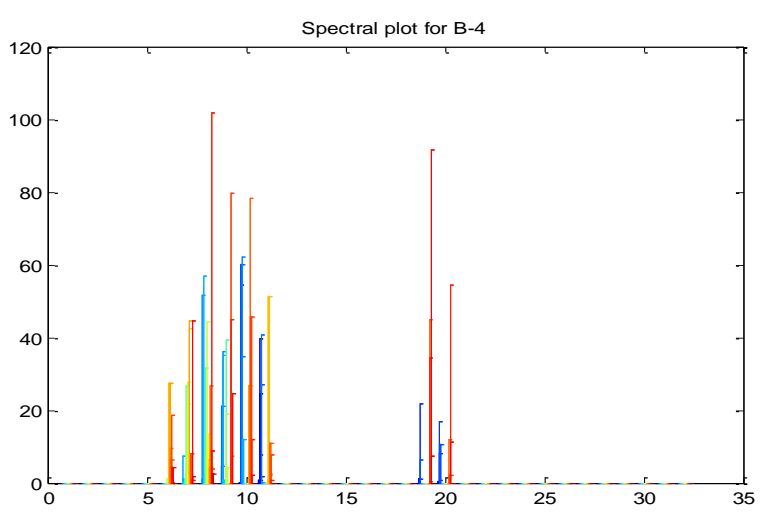

Fig. 17: Histogram plot for the query 


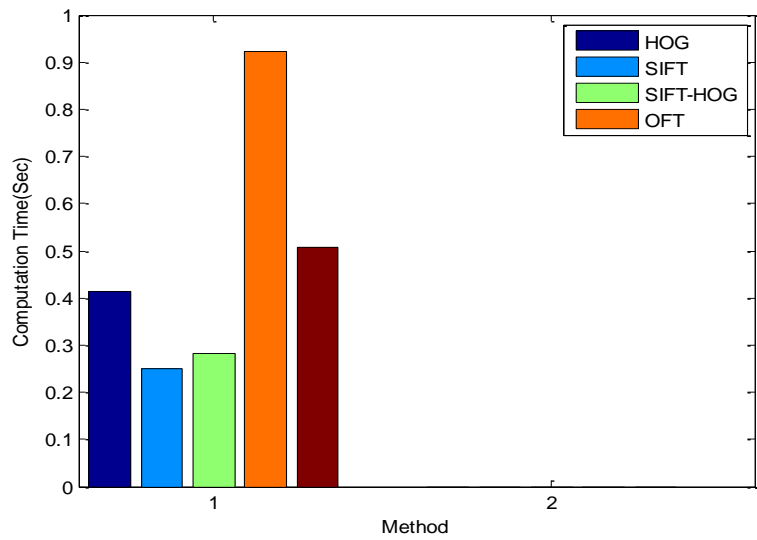

Fig. 18: Computation time plot

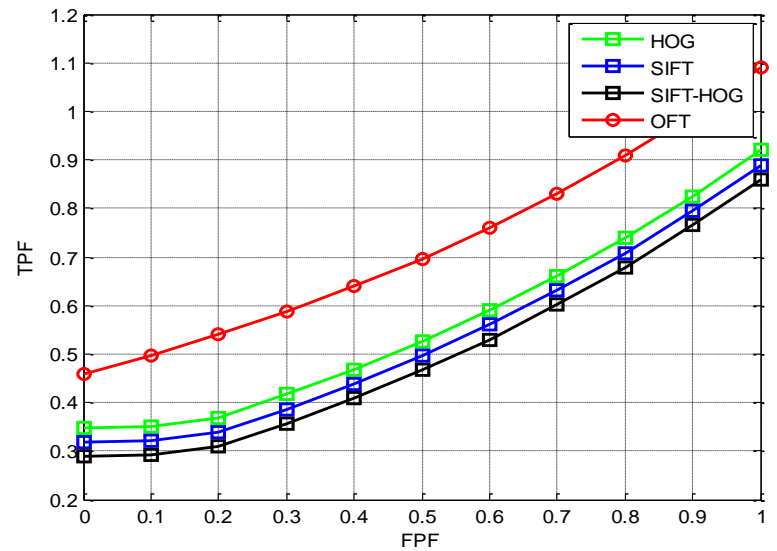

Fig. 19: ROC curve

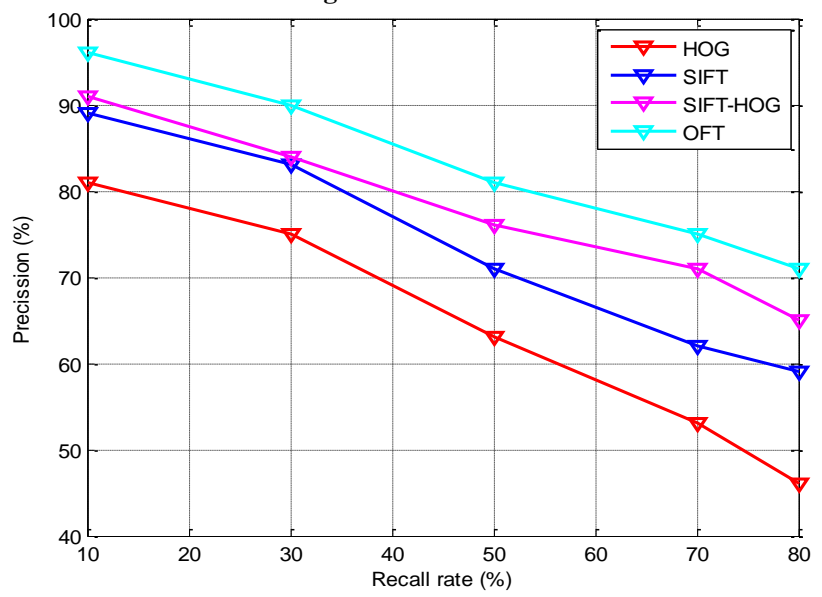

Fig. 20: Precision plot
For the evaluation of the proposed approach a image retrieval performance defined by the true positive (TP), true negative (TN), false positive (FP), and false negative (FN) defining accuracy, sensitivity, precision etc., were used. The performance metrics for the evaluation were derived as,

Accuracy $=\frac{T P+T N}{T P+T N+F P+F N}$

This suggested approach for its evaluation, developed the parameters of sensitivity, specificity, recall, precision and Fmeasure for its evaluation analysis using the measured parameters. To compute the measured parameters, a mathematical expressions used are defined as, evaluation were derived as,

Sensitivity $=\frac{T P}{T P+F N}$

The specificity is given by,

Specificity $=\frac{T N}{T N+F P}$

The recall and the precision is given by,

$$
\begin{aligned}
& \text { Recall }=\frac{T P}{T P+F N} \\
& \text { Precision }=\frac{T P}{T P+F P} \\
& F \_ \text {measure }=\frac{2 * \text { Recall.Precision }}{\text { Recall }+ \text { Precision }}
\end{aligned}
$$

Table 1: Performance Evaluation for the Developed Test Model

\begin{tabular}{|c|c|c|c|c|c|c|c|c|}
\hline Test Sample & Feature Selection & Accuracy & Sensitivity & Specificity & Recall & Precision & F-Measure & Time \\
\hline \multirow{4}{*}{ Sample-1 } & SIFT & 57.670 & 0.220 & 0.608 & 0.220 & 0.680 & 0.478 & 0.545 \\
\cline { 2 - 8 } & HOG & 63.500 & 0.415 & 0.752 & 0.315 & 0.740 & 0.523 & 0.348 \\
\cline { 2 - 8 } & SIFT-HOG & 71.000 & 0.544 & 0.799 & 0.444 & 0.800 & 0.571 & 0.138 \\
\cline { 2 - 8 } & OFT & 78.127 & 0.668 & 0.899 & 0.714 & 0.952 & 0.796 & 0.110 \\
\hline \multirow{4}{*}{ Sample-2 } & SIFT & 49.484 & 0.432 & 0.712 & 0.432 & 0.508 & 0.542 & 0.273 \\
\cline { 2 - 8 } & HOG & 58.134 & 0.458 & 0.854 & 0.458 & 0.666 & 0.621 & 0.143 \\
\cline { 2 - 8 } & SIFT-HOG & 69.500 & 0.524 & 0.946 & 0.524 & 0.820 & 0.652 & 0.137 \\
\cline { 2 - 8 } & OFT & 75.210 & 0.745 & 0.991 & 0.785 & 0.889 & 0.733 & 0.124 \\
\hline
\end{tabular}

\section{Conclusion}

The proposed approach of image retrieval using sketch is proposed. The image retrieval system is defined with a orientation feature descriptor to validate the feature of low dimensionality by introducing a average orientation to the Gabor filter. The obtained results of histogram representation are normalized by the averaging model, and a threshold value is derived based on the neighboring correlative relation to minimize the processing overhead. The retrieval performance of the developed approach illustrates the application of SBIR on coil-100 data base. The performance observed w.r.t. accuracy and time metric validates the efficiency of the proposed approach. 


\section{References}

[1] Y H Sharath Kumara, D S Gurub, "Retrieval of Sample Based on Sketches”, Procedia Computer Science, Elsevier, vol.46, 1577 1584, 2015.

[2] Jun Xiao a, Zhang peng Tang a,n, YinfuFeng a, Zhidong Xiao , "Sketch-based human motion retrieval via selected 2D geometric posture descriptor" , Published by Elsevier B.V, Signal Processing, 2015

[3] Ana Sofia Mouratoa and Rui Jesus, "Clip art retrieval using a sketch Tablet application", Conference on Electronics, Telecommunications and Computers - CETC 2013.

[4] NadiahYusof, TengkuSitiMeriamTengkuWook, SitiFadzilah Ma Noor, "Songket Motives Retrieval Through Sketching Technique", The 4th International Conference on Electrical Engineering and Informatics (ICEEI 2013). Published by Elsevier B.V.

[5] Dipika R. Birari1, Prof. J.V. Shinde, "Survey on Sketch Based Image Retrieval", International Journal of Advanced Research in Computer and Communication Engineering Vol. 4, Issue 12, December 2015

[6] Gayathri Devi K, "A Survey on Sketch Based Image Retrieval", Elk Asia Pacific Journal Of Computer Science And Information Systems, Volume 1, Issue 2, 2015.

[7] NadiahYusof, TengkuSitiMeriamTengkuWook, SitiFadzilah Ma Noor, "Songket Motives Retrieval Through Sketching Technique", The 4th International Conference on Electrical Engineering and Informatics (ICEEI 2013)

[8] Yi Li , Yi-Zhe Song, Shaogang Gong, "Fine-Grained SketchBased Image Retrieval by Matching Deformable Part Models", Timothy M. Hospedales.

[9] Rong Zhou, Liuli Chen and Liqing Zhang, "Sketch-based Image Retrieval on a Large Scale Database", MM'12, October 29November 2, 2012.

[10] Prachi A. Gaidhani, S.B. Bagal, "Implementation of Sketch Based and Content Based Image Retrieval", Department of Electronics \& Telecommunication.

[11] R. Aarthi1, K. P. Anjana1 and J. Amudha, "Sketch based Image Retrieval using Information Content of Orientation" , Indian Journal of Science and Technology, Vol 9(1).

[12] Yusuke Matsui, KiyoharuAizawa, Yushi Jing, "SKETCH 2 MANGA: Sketch-Based Manga Retrieval", the University of Tokyo.

[13] Peng Xu1, Qiyue Yin2, Yonggang Qi1, Yi-Zhe Song, "InstanceLevel Coupled Subspace Learning for Fine-Grained Sketch-Based Image Retrieval" ,Springer International Publishing Switzerland 2016.

[14] YonggangQiy Yi-Zhe Song, HonggangZhangy Jun Liu, "sketchbased image retrieval via siamese convolution neural network", School of Information and Communication Engineering, BUPT, Beijing, China

[15] Mathias Eitz, Kristian Hildebrand, TamyBoubekeur and Marc Alex, "A descriptor for large scale image retrieval based on sketched feature lines",Eurographics Symposium on Sketch-Based Interfaces and Modeling (2009).

[16] Rui Hu, Tinghuai Wang, and John Collomosse, "A Bag-OfRegions Approach To Sketch-Based Image Ret" , Centre for Vision, Speech and Signal Processing University of Surrey, Guildford, Surrey, UK.

[17] Avinash Yadlapati, Dr. Hari Kishore Kakarla, "An Advanced AX Protocol Verification using Verilog HDL", Wulfenia Journal, ISSN: 1561-882X, Volume 22, Number 4, pp. 307-314, April 2015

[18] P Ramakrishna, K. Hari Kishore, "Design of Low Power 10GS/s 6-Bit DAC using CMOS Technology "International Journal of Engineering and Technology(UAE), ISSN No: 2227-524X, Vol No: 7, Issue No: 1.5, Page No: 226-229, January 2018

[19] A Murali, K. Hari Kishore, "Efficient and High Speed Key Independent AES Based Authenticated Encryption Architecture using FPGAs "International Journal of Engineering and Technology(UAE), ISSN No: 2227-524X, Vol No: 7, Issue No: 1.5, Page No: 230-233, January 2018.

[20] G.S.Spandana, K Hari Kishore "A Contemporary Approach For Fault Diagnosis In Testable Reversible Circuits By Employing The CNT Gate Library" International Journal of Pure and Applied Mathematics, ISSN No: 1314-3395, Vol No: 115, Issue No: 7, Page No: 537-542, September 2017

[21] K Hari Kishore, CVRN Aswin Kumar, T Vijay Srinivas, GV Govardhan, Ch Naga Pavan Kumar, R Venkatesh "Design and
Analysis of High Efficient UART on Spartran-6 and Virtex-7 Devices", International Journal of Applied Engineering Research, ISSN 0973-4562, Volume 10, Number 09 , pp. 23043-23052, June 2015

[22] K Bindu Bhargavi, K Hari Kishore "Low Power BIST on Memory Interface Logic", International Journal of Applied Engineering Research, ISSN 0973-4562, Volume 10, Number 08, pp. 21079 21090, May 2015

[23] Korraprolu Brahma Reddy, K Hari Kishore, "A Mixed Approach for Power Dissipation Reduction in Nanometer CMOS VLSI circuits", International Journal of Applied Engineering Research, ISSN 0973-4562 Volume 9, Number 18 , pp. 5141-5148, July 2014.

[24] Nidamanuri Sai Charan, Kakarla Hari Kishore "Reorganization of Delay Faults in Cluster Based FPGA Using BIST" Indian Journal of Science and Technology, ISSN No: 0974-6846, Vol No.9, Issue No.28, page: $1-7$, July 2016

[25] Sravya Kante, Hari Kishore Kakarla, Avinash Yadlapati,"Design and Verification of AMBA AHB-Lite protocol using Verilog HDL" International Journal of Engineering and Technology, EISSN No: 0975-4024, Vol No.8, Issue No.2, Page:734-741, May 2016

[26] Bandlamoodi Sravani, K Hari Kishore, "An FPGA Implementation of Phase Locked Loop (PLL)", International Journal of Applied Engineering Research, ISSN 0973-4562, Volume 10, Number 14, pp. 34137-34139, August 2015.

[27] Avinash Yadlapati, Kakarla Hari Kishore,"Constrained Level Validation of Serial Peripheral Interface Protocol", Proceedings of the First International Conference on SCI 2016, Volume 1, Smart Computing and Informatics, Smart Innovation, Systems and Technologies 77, ISSN No: 2190-3018, ISBN: 978-981-10-55447, Chapter No: 77, pp. 743-753, 25 $5^{\text {th }}$ December 2017.

[28] [28] P Kiran Kumar, P Prasad Rao, Kakarla Hari Kishore, "Optimal Design of Reversible Parity Preserving New Full Adder / Full Subtractor", IEEE SPONSORED 3rd INTERNATIONAL CONFERENCE ON ELECTRONICS AND COMMUNICATION SYSTEMS (ICECS 2016), pp. $3465-3470,25^{\text {th }}$ and $26^{\text {th }}$ February 2016.

[29] Y Avinash, K Hari Kishore 'DDesigning Asynchronous FIFO for Low Power DFT Implementation'” International Journal of Pure and Applied Mathematics, ISSN No: 1314-3395, Vol No: 115 Issue No: 8, Page No: 561-566, September 2017

[30] Mahesh Mudavath and K Hari Kishore "Design of RF Front End CMOS Cascade CS Low Noise Amplifier on 65nm Technology Process" International Journal of Pure and Applied Mathematics, ISSN No: 1314-3395, Vol No: 115, Issue No: 7, Page No: $417-$ 422, September 2017

[31] P. Sahithi K Hari Kishore, E Raghuveera, P. Gopi Krishna "DESIGN OF VOLTAGE LEVEL SHIFTER FOR POWEREFFICIENT APPLICATIONS USING 45nm TECHNOLOGY" International Journal of Engineering and Technology(UAE), ISSN No: 2227-524X, Vol No: 7, Issue No: 2.8, Page No: 103-108, March 2018.

[32] N Bala Dastagiri K Hari Kishore "A 14-bit 10kS/s Power Efficient $65 \mathrm{~nm}$ SAR ADC for Cardiac Implantable Medical Devices" International Journal of Engineering and Technology(UAE), ISSN No: 2227-524X, Vol No: 7, Issue No: 2.8, Page No: 34-39, March 2018.

[33] S.V.Manikanthan and T.Padmapriya "Recent Trends In M2m Communications In 4g Networks And Evolution Towards 5g", International Journal of Pure and Applied Mathematics, ISSN NO: 1314-3395, Vol-115, Issue -8, Sep 2017.

[34] S.V. Manikanthan, T. Padmapriya "An enhanced distributed evolved node-b architecture in 5G tele-communications network" International Journal of Engineering \& Technology (UAE), Vol 7 Issues No (2.8) (2018) 248-254.March2018.

[35] S.V. Manikanthan, T. Padmapriya, Relay Based Architecture For Energy Perceptive For Mobile Adhoc Networks, Advances and Applications in Mathematical Sciences, Volume 17, Issue 1, November 2017, Pages 165-179 